\title{
Classification of TM-Algebra
}

\author{
K.Megalai \\ Department of Mathematics \\ Bannari Amman Institute of Technology \\ Sathyamangalam, Erode Dist. \\ Tamil Nadu , India
}

\author{
Dr.A.Tamilarasi \\ Kongu Engineering College \\ Perundurai, Erode Dist. \\ Tamil Nadu , India
}

\begin{abstract}
In this paper, the notion of TM-algebra is introduced which is a generalization of $\mathrm{Q} / \mathrm{BCI} / \mathrm{BCH}$-algebras. The concepts of positive implicative, implicative, 1-weakly positive implicative, 2-weakly positive implicative, right translation, left translation, weak right translation, weak left translation and the related properties are duly characterized.
\end{abstract}

\section{Mathematics Subject Classification: 06F 35, 03G 25}

\section{Keywords}

TM-Algebra, implicatives, weakly and positive implicatives, right and left translation.

\section{INTRODUCTION}

In 1966, Imai and Iseki introduced two classes of abstract algebras, BCK-algebras and BCI-algebras. These algebras have been extensively studied since their introduction. In 1983, $\mathrm{Hu}$ and $\mathrm{Li}$ introduced the notion of a $\mathrm{BCH}$-algebra which is a generalization of the notion of BCK and BCI-algebras and studied a few properties of these algebras. After that J.Neggers, S.S.Ahn and H.S.Kim introduced Q-algebras which is a generalization of $\mathrm{BCK} / \mathrm{BCI}$-algebras and obtained several results.

Here, a new notion of TM-algebra is introduced, which is a generalization of $\mathrm{Q} / \mathrm{BCK} / \mathrm{BCI} / \mathrm{BCH}$-algebras. The oncepts of weakly commutative, positive implicative, implicative, weakly positive implicative and weakly implicative were introduced in $\mathrm{BCH}$-algebra. In this note, the characterization of positive implicative, implicative, 1- weakly positive implicative, 2-weakly positive implicative, weak right translation, weak left translation are introduced.

\section{PRELIMINARIES}

In this section, certain definitions, known results and examples that will be used in the sequel are described..

\subsection{Definition}

A BCI-algebra is an algebra $(X, *, 0)$ of type $(2,0$ ) satisfying the following conditions:

i) $(x * y) *(x * z) \leq z * y$

ii) $x *(x * y) \leq y$

iii) $x \leq x$,

iv) $x \leq y$ and $y \leq x$ imply $x=y$

v) $x \leq 0$ implies $x=0$, where $x \leq y$ is defined by $x * y=0$ for all $x, y, z \in X$.

\subsection{Definition}

A BCK-algebra is an algebra $\left(X,{ }^{*}, 0\right)$ of type $(2,0$ ) satisfying the following conditions:

i) $(x * y) *(x * z) \leq z * y$

ii) $x *(x * y) \leq y$

iii) $x \leq x$,

iv) $x \leq y$ and $y \leq x$ imply $x=y$,

v) $0 \leq x$ implies $x=0$, where $x \leq y$ is defined by $x * y=0$ for all $x, y, z \in X$.

\subsection{Definition}


A BCH-algebra is an algebra $(X, *, 0)$ of type $(2,0$ ) satisfying the following conditions :

i) $x * x=0$

ii) $(x * y) * z=(x * z) * y$.

iii) If $x * y=0$ and $y * x=0$ imply $x=y$, for all $x, y, z \in$ $X$.

\subsection{Definition}

A Q-algebra is an algebra $(\mathrm{X} * 0)$ of type $(2,0)$ satisfying the following axioms :

i) $x * x=0$

ii) $x * 0=x$

iii) $(x * y) * z=(x * z) * y$, for all $x, y, z \in X$.

Every BCK-algebra is a BCI-algebra but not conversely.

Every BCI-algebra is a $\mathrm{BCH}$-algebra but not conversely

Every $\mathrm{BCH}$-algebra is a Q-algebra but not conversely.

Every Q-algebra satisfying the conditions

$(x * y) *(x * z)=z * y$ and $x * y=0, y * x=0$ imply $x=y$

is a BCI-algebra.

\section{TM-ALGEBRA}

\subsection{Definition}

A TM-algebra $(X, *, 0)$ is a non-empty set $X$ with a constant " 0 " and a binary operation "* " satisfying the following axioms :

i) $x * 0=x$

ii) $(x * y) *(x * z)=z * y$ for any $x, y, z \in X$

In $X$ we can define a binary relation $\leq$ by $x \leq y$ if and only if $x * y=0$

In any TM-algebra $(X, *, 0)$, the following holds good for all $x, y, z \in X$

iii) $x * x=0$

iv) $(x * y) * x=0 * y$

v) $x *(x * y)=y$ vi) $(x * z) *(y * z) \leq x * y$

vii) $(x * y) * z=(x * z) * y$

viii) $x * 0=0 \Rightarrow x=0$

xi) $x \leq y \Rightarrow x * z \leq y * z$ and $z * y \leq z * x$

x) $\quad x *(x *(x * y))=x * y$

xi) $0 *(x * y)=y * x=(0 * x) *(0 * y)$

xii) $(x *(x * y)) * y=0$

xiii) If $x * y=0, y * x=0$ then $x=y$.

A QS-algebra is obviously a TM -algebra, But a TM-algebra is said to be a QS-algebra if it satisfies the additional relations $(x * y) * z=(x * z) * y$ and $y * z=z * y$ for all $x, y, z \in X$.

\subsection{Example}

Let $X=\{0,1,2,3\}$ be a set with cayley table ( Table 1 )

\section{Table 1}

\begin{tabular}{c|cccc}
$*$ & 0 & 1 & 2 & 3 \\
\hline 0 & 0 & 1 & 2 & 3 \\
1 & 1 & 0 & 3 & 2 \\
2 & 2 & 3 & 0 & 1 \\
3 & 3 & 2 & 1 & 0
\end{tabular}

Then $(X, *, 0)$ is a TM- algebra .

The relations between TM-algebra and other algebras are investigated and presented below.

\subsection{Theorem}

Every BCK- algebra is a TM-algebra but the converse is not true.

The above example 3.2 is a TM - algebra but not BCK-algebra, since $0 * x \neq 0$ for all $x=1,2,3$.

\subsection{Theorem}

Every TM -algebra is a $\mathrm{BH}$ - algebra, but the converse is not true. Similarly, every TM- algebra is a Q-algebra (Table $2)$, but the converse is not true. 


\subsection{Example}

Let $X=\{0,1,2,3\}$

Table 2

\begin{tabular}{c|cccc}
$*$ & 0 & 1 & 2 & 3 \\
\hline 0 & 0 & 0 & 0 & 0 \\
1 & 1 & 0 & 0 & 0 \\
2 & 2 & 0 & 0 & 0 \\
3 & 3 & 3 & 3 & 0
\end{tabular}

Note that $(X, *, 0)$ is a Q-algebra .

The condition $(x * y) *(x * z)=z * y$ is not satisfied as $(1 * 2) *(1 * 3)=0 * 0=0 \neq 3=3 * 2$

\subsection{Theorem}

Every TM-algebra is a $\mathrm{BCH}$-algebra. Every $\mathrm{BCH}$ algebra satisfying $(x * y) *(x * z)=z * y$ is a TM-algebra .

\subsection{Theorem}

Every TM- algebra $\mathrm{X}$ satisfying $x * z=z$ is a trivial algebra.

\subsection{Definition}

Let $(X, *, 0)$ be a TM -algebra. A non-empty subset $I$ of $X$ is called an ideal of $X$ if it satisfies

(i) $0 \in I$

ii) $x * y \in I$ and $y \in I$ imply $x \in I$ for all $x, y \in X$.

Any ideal $I$ has the property that $y \in I$ and $x \leq y$ imply $x \in I$

\subsection{Example}

Table-3

\begin{tabular}{c|cccc}
$*$ & 0 & $a$ & $b$ & $c$ \\
\hline 0 & 0 & 0 & $c$ & $b$ \\
b & a & o & $c$ & $b$ \\
$c$ & $c$ & $b$ & o & $c$ \\
& c & $c$ & $b$ & 0
\end{tabular}

Then the set $I=\{0, a\}$ is an ideal of $X$.

\section{CLASSIFICATION OF TM-ALGEBRA.}

\subsection{Definition}

A BCK-algebra $(X, *, 0)$ is called positive implicative

if $(x * y) * z=(x * z) *(y * z)$, for all $x, y, z \in X$..

It is called implicative if $x *(y * x)=x$. It is commutative if $x *(x * y)=y *(y * x)$, for all $x, y \in X$.

\subsection{Definition}

A BCI-algebra $(X, *, O)$ is called weakly positive implicative if $(x * y) * z=((x * z) * z) *(y * z)$ for all $x, y, z \in X$

It is called weakly implicative if

$(x *(y * x)) *(0 *(y * x))=x$. for all $x, y \in X$.

It is weakly commutative if

$(x *(x * y)) *(0 *(x * y))=y *(y * x)$ for all $x, y \in$ $X$.

\subsection{Theorem}

A BCI-algebra $(X, *, 0)$ is called weakly positive implicative if and only if $x * y=((x * y * y) *(0 * y)$.

\subsection{Definition}

A $\mathrm{BCH}$-algebra $(\mathrm{X}, *, 0)$ is called weakly positive implicative if $x * y=((x * y) * y) *(0 * y)$ for all $x, y \in X$.

It is called weakly implicative if $(x *(y * x)) *(0 *(y * x))=x$ for all $x, y \in X$.

It is weakly commutative if

$(x *(x * y)) *(0 *(x * y))=y *(y * x)$, for all $x, y \in X$.

Every weakly implicative BCI-algebra $X$ is a weakly positive implicative BCI-algebra.

Every implicative BCK-algebra $X$ is a positive implicative BCK-algebra. 


\subsection{Definition}

A TM-algebra $(X, *, 0)$ is called positive implicative if $(x * y) * z=(x * z) *(y * z)$ for all $x, y, z \in X$.

It is called implicative if $x *(y * x)=x$.

\subsection{Definition}

An ideal $I$ of a TM-algebra $(X, *, 0)$ is said to be implicative if $(x * y) * z \in I$ and $y * z \in I$ imply $x * z \in I$ for any $x, y, z \in X$

\subsection{Theorem}

Let $(X, *, 0)$ be a TM-algebra and let $I$ be an implicative ideal of $X$. Then $G(X) \subseteq I$.

\section{Proof}

Let $x \in G(X)$. Then $0 * x=x$.

Now $0=x * x=(0 * x) * x \in I$ and $x * x=0 \in I$.

Since $I$ is an implicative ideal $0 * x \in I$.

That is $x \in I$.

Hence $G(X) \subseteq I$.

\subsection{Definition}

A TM-algebra $(X, *, 0)$ is said to be 1 -weakely positive implicative if

$((x * y) * y) *(0 * y)=x * y$, for all $x, y \in X$.

A TM-algebra $(X, *, 0)$ is said to be 2-weakely

positive implicative if

$$
(x *(x * y)) *(0 *(x * y))=x * y, \text { for all } x, y \in X
$$

\subsection{Definition}

Let $(X, *, 0)$ be a TM-algebra. For a fixed $\mathrm{x} \in X$, the map $R_{x}: X \rightarrow X$ given by $R_{x}(y)=y * x$, for all $y \in X$ is called a right translation of $X$.

Similarly, the map $R_{x}^{\prime}: X \rightarrow X$ given by $R_{x}^{\prime}(y)=x * y$, for all $y \in X$, is called a left translation of $X$.

\subsection{Definition}

Let $(X, *, 0)$ be a TM-algebra. For a fixed $x \in X$ the map $T_{x}: X \rightarrow X$ given by $T_{x}(y)=(y * x) *(0 * x)$, for all $y$ $\in X$, is called a weak right translation of $X$.

Similarly the map $T_{x}{ }^{\prime}: X \rightarrow X$ given by $T_{x}{ }^{\prime}(y)=(x * y) *(0 * y)$, for all $y \in x$ is called a weak left translation of $X$.

The following theorem characterizes the weakly positive implicative TM-algebra

\subsection{Theorem}

A TM-algebra $(X, *, 0)$ is 1 -weakly positive implicative if and only if $R_{z}=T_{z} \circ R_{z}$ for all $z \in X$, and " $O$ " is the composition of functions.

\section{Proof}

Let $X$ be a TM-algebra and let $R_{z}=T_{z} \circ R_{z}$ for $z$

$\in X$. Then

$$
\begin{aligned}
y * z=R_{z}(y) & =\left(T_{z} \circ R_{z}\right)(y) \\
& =T_{z} \circ\left(R_{z}(y)\right) \\
& =T_{z}(y * z) \\
& =((y * z) * z) *(0 * z), \text { for all } y, z \in X .
\end{aligned}
$$

Hence $X$ is 1-weakly positive implicative TM-algebra. Conversely, if $X$ is a 1-weakly positive implicative TM-algebra then

$$
\begin{aligned}
& (y * z)=((y * z) * z) *(0 * z) \\
& \text { So } \quad \begin{aligned}
R_{z}(y) & =\mathrm{y} * \mathrm{z} \\
& =((y * z) * \mathrm{z}) *(0 * z) \\
& =\left(\left(R_{z}(y) * \mathrm{z}\right) *(0 * \mathrm{z})\right. \\
& =T_{z}\left(R_{z}(y)\right) \\
& =\left(T_{z} \circ R_{\mathrm{z}}\right)(y), \text { for all } y, z \in X .
\end{aligned}
\end{aligned}
$$

Hence $R_{z}=T_{z} \circ R_{z}$

\subsection{Theorem}

A TM-algebra $X$ is 2 weakly positive implicative iff $R_{z}^{\prime}=T_{z^{\prime}} \circ R_{z^{\prime}}$ for all $z \in X$ 


\section{Proof}

Let $X$ be a TM-algebra and let $R_{z}{ }^{\prime}=T_{z}{ }^{\prime} \circ R_{z}{ }^{\prime}$ for $z \in X$.

Then

$$
\begin{aligned}
z^{*} y & =R_{z^{\prime}}(y) \\
& =\left(T_{z^{\prime}} \circ R_{z^{\prime}}\right)(y) \\
= & T_{z^{\prime}}\left(R_{z^{\prime}}(y)\right) \\
= & T_{z^{\prime}}\left(z^{*} y\right) \\
= & \left(z *\left(z^{*} y\right) *(0 *(z * y)) \text { for all } y, z \in X .\right.
\end{aligned}
$$

Hence $X$ is 2-weakly positive implicative TM-algebra.

Conversely,

if $X$ is 2-weakly positive implicative TM-algebra, then

$$
x * y=(x *(x * y)) *(0 *(x * y))
$$

Now,

$$
\begin{aligned}
R_{x}^{\prime}(y) & =x * y \\
& =(x *(x * y)) *(0 *(x * y)) \\
& =\left(x * R_{x}^{\prime}(y)\right) *\left(0 * R_{x}^{\prime}(y)\right) \\
& =T_{x}^{\prime}\left(R_{x}^{\prime}(y)\right) \\
& =\left(T_{x}{ }^{\prime o} R_{x}^{\prime}\right)(y) \text { for all } x, y, \in X .
\end{aligned}
$$

Hence $R_{x}^{\prime}=T_{x}^{\prime} \circ R_{x}^{\prime}$.

\subsection{Theorem}

Let $X$ be a 1-weakly positive implicative TM-algebra.

Then $T_{y}=T_{y} \circ T_{y}=T_{y}^{2}$

\section{Proof}

Let $X$ be a 1-weakly positive implicative TM-algebra.

Then

$x * y=((x * y) * y) *(0 * y)$.

Now

$$
\begin{aligned}
(x * y) *(0 * y)=((x * y) * y) *(0 * y)) *(0 * y) & =(((x * y) *(0 * y)) * y) *(0 * y) . \\
T_{y}(x) & =(x * y) *(0 * y) \\
T_{y}{ }^{2}(x) & =T_{y}((x * y) *(0 * y))
\end{aligned}
$$

$$
\begin{aligned}
& =(((x * y) *(0 * y)) * y) *(0 * y) \\
& =(x * y) *(0 * y) \\
& =T_{y}(x), \text { for all } x, y, \in X .
\end{aligned}
$$

The converse of the above theorem is not true, which can be shown by the following example (Table 3 ).

Let $X=\{0, \mathrm{a}, \mathrm{b}, \mathrm{c}\}$ in which $*$ is defined by the following table.

\section{Table 3}

\begin{tabular}{c|cccc}
$*$ & 0 & $\mathrm{a}$ & $\mathrm{b}$ & $\mathrm{c}$ \\
\hline 0 & 0 & 0 & $\mathrm{~b}$ & $\mathrm{~b}$ \\
$\mathrm{a}$ & $\mathrm{a}$ & $\mathrm{o}$ & $\mathrm{b}$ & $\mathrm{b}$ \\
$\mathrm{b}$ & $\mathrm{b}$ & $\mathrm{b}$ & $\mathrm{o}$ & 0 \\
$\mathrm{c}$ & $\mathrm{c}$ & $\mathrm{b}$ & $\mathrm{a}$ & 0
\end{tabular}

Then $(X, *, 0)$ is a $\mathrm{BCI}$ algebra, which in turn is a TM-algebra.

As $a=c * b \neq((c * b) * b) *(0 * b)=(a * b) *(0 * b)=$ $b * b=0, X$ is not 1-weakly positive implicative .

But $T_{0}^{2}=T_{0}, T_{a}^{2}=T_{a}, \quad T_{b}^{2}=T_{b}, \quad T_{c}{ }^{2}=T_{c}$

\subsection{Theorem}

Let $X$ be a 2- weakly positive implicative TM-algebra. Let $T_{x}{ }^{\prime}(y) \in G(X)$ and $x \leq T_{x}{ }^{\prime}(y)$. Then $T_{x}{ }^{\prime 2}=T_{x}{ }^{\prime 2}$ for all $x, y, \in X$.

\section{Proof}

Let $X$ be a 2-weakly positive implicative TM-algebra.

Then $(x *(x * y)) *(0 *(x * y))=x * y$

Now

$T_{x}^{\prime 2}(y)=T_{x}^{\prime}\left(T_{x}^{\prime}(y)\right)$

$$
\begin{aligned}
& =T_{x}^{\prime}((x * y) *(0 * y)) \\
& =(x *((x * y) *(0 * y))) *(0 *((x * y) *(0 *
\end{aligned}
$$

$y)))$

$$
\begin{aligned}
& =0 *((x * y) *(0 * y)) \text { since } x \leq T_{x}^{\prime}(y) \\
& =(x * y) *(0 * y), \text { since } T_{x}^{\prime}(y) \in G(X) \\
& =T_{x}^{\prime}(y)
\end{aligned}
$$

Converse need not be true. 


\section{REFERENCES}

[1] M.A.Chaudhry. Weakly positive implicative and weakly implicative BCI-algebra, Math.Japon. 35(1990), no.1.141-151. MR 90k:06019.Zbl 702.06014.

[2] M.A.Chaudhry and Hafiz Fakhar-Ud-Din. On Some classes of BCH- algebras, IJMMS 25:3 (2001) , PII S0161171201003957.

[3] Q.P. Hu and X. Li. On BCH-algebras, Math. Sem. Notes Kobe Univ. 2 (1983), 313-320.

[4] Q.P. Hu and X. Li, On proper BCH-algebras, Math. Japon. 30 (1985), 659- 661.

[5] Y.Imai and K.Isaeki. On axiom systems of propositional calculi, XIV, Proc.Japan Acad.42 (1996), 19-22. MR 33\# 3902. Zbl 156.24812
[6] K.Iseki and S.Tanaka, An introduction to the theory of BCK-algebras, Math. Japon.23 ( 1978/79 ), no.1, 1-26 MR 80a:03081.zbi 385.0305.

[7] K. Iseki, On BCI -algebras, Math. Sem. Notes Kobe Univ. 8 (1980), 25- 130.

[8] J. Neggers, S. S. Ahn and H. S. Kim, On Q-algebras, IJMMS 27 (2001), 749-757.

[9] Tao Sun, Dahai Hu and Xiquan Liang, Several classes of BCK-algebras and their properties, Formalized Mathematics 2007, vol.15. 